DESAFÍOS ACTUALES A LA INCLUSIÓN: UN ESTUDIO DE CASO EN UN AULA DE PREESCOLAR PORTUGUESA

TODAY'S ISSUES TO THE INCLUSION: A CASE STUDY IN A EARLY CHILDHOOD EDUCATION PORTUGUESE CLASSROOMS

\author{
Volumen 15, Número 1 \\ Enero - Abril \\ pp. 1-18
}

Este número se publicó el $1^{\circ}$ de enero de 2015

DOI: dx.doi.org/10.15517/aie.v15i1.17736

\author{
Maㅡ Esther Martínez-Figueira \\ $\mathrm{M}^{\mathrm{a}}$ Beatriz Páramo-Iglesias \\ Eugénia Maria de Matos Claudino Necho
}

Revista indizada en REDALYC, SCIELO

Revista distribuida en las bases de datos:

CATÁLOGO DE LATINDEX, IRESIE, CLASE, DIALNET, DOAJ, E-REVIST@S, SHERPA/ROMEO, QUALIS, MIAR

Revista registrada en los directorios:

ULRICH'S, REDIE, RINACE, OEI, MAESTROTECA, PREAL, $\underline{\text { CLACSO }}$ 


\title{
DESAFÍOS ACTUALES A LA INCLUSIÓN: UN ESTUDIO DE CASO EN UN AULA DE PREESCOLAR PORTUGUESA \\ TODAY'S ISSUES TO THE INCLUSION: A CASE STUDY IN A EARLY CHILDHOOD EDUCATION PORTUGUESE CLASSROOMS
}

\author{
$M^{a}$ Esther Martínez-Figueira ${ }^{1}$ \\ $M^{a}$ Beatriz Páramo-Iglesias ${ }^{2}$ \\ Eugénia Maria de Matos Claudino Necho ${ }^{3}$
}

\begin{abstract}
Resumen: Este ensayo muestra una reflexión sobre el nuevo enfoque de la escuela del presente y del futuro en las aulas portuguesas, la cual es necesaria para el proceso de cambio hacia una educación inclusiva. Afrontamos esta tarea con el objetivo de valorar las percepciones acerca de la educación inclusiva que realiza una maestra de un Jardín de Infancia ubicado en Vila Real (Portugal). Para ello llevamos a cabo, en el curso escolar 2013/2014, una investigación de corte cualitativo e interpretativo basada en un estudio de caso de realidad única con un marcado carácter descriptivo y exploratorio para confirmar la emergencia pedagógica que apuesta por la educación inclusiva. La toma de datos se ejecuta por una combinación de instrumentos (entrevista, escala de observación, documentos, materiales audiovisuales y notas de campo) y fuentes de información (docente e investigadoras) con la finalidad de realizar una triangulación de datos y poder, de esta manera, confirmar la emergencia de un ideario y prácticas docentes inclusivas en esta aula. Los primeros resultados nos ofrecen varios puntos para la reflexión: (1) una mirada a la inclusión, a la equidad, en términos de oportunidad, de plantearnos retos y desafíos; (2) se sugiere la necesidad de llevar a cabo cambios en las prácticas y valores docentes; $y$ (3) se entiende como algo natural que los espacios, actividades, tiempos y relaciones respondan a un conjunto de elementos que, según unas u otras combinaciones, se configuren para hacer efectiva la realidad inclusiva en el aula a la que se refiere la propuesta, únicamente a lo particularizante del estudio, sin pretender hacer generalizaciones.
\end{abstract}

Palabras clave: EDUCACIÓN INCLUSIVA, PRIMERA INFANCIA, EVALUACIÓN DOCENTE, ESTUDIO DE CASO, INCLUSIÓN, PORTUGAL.

\begin{abstract}
This essay is a reflection on the new paradigm of school nowadays in Portuguese classrooms, required to the process of change towards inclusive education. We face this task with the aim of assessing a kindergarten teacher's inclusive perceptions in Vila Real (Portugal) in the school year 2013/2014. It is a qualitative and interpretative research from a descriptive and exploratory perspective based on a case study. In order to collect Data collection it was performed by a triangulation of instruments (interview, observation scale, documents, audiovisual materials and field notes) and different information sources (teacher and researchers), in order to perform a triangulation of data and to confirm the emergence of an inclusive ideology and teaching practices in this classroom. First results provide several points for reflection: (1) a look at the inclusion, equity, in terms of opportunity, to ask issues and challenges; (2) it is suggested the need to make changes in teaching practices and values; and (3) it's something natural that this whole elements (spaces, activities, times and relationships) are configured to realize an inclusive education in classroom the proposal relates, only to the particularizing of the study, without trying to make generalizations.
\end{abstract}

Key words: INCLUSIVE EDUCATION, EARLY CHILDHOOD, TEACHER EVALUATION, CASE STUDY, INCLUSION, PORTUGAL.

\footnotetext{
${ }^{1}$ Desarrolla su actividad docente, investigadora y de gestión en la Facultad de Ciencias de la Educación y del Deporte de la Universidad de Vigo. España. Dirección electrónica: esthermf@uvigo.es

2 Personal Docente e Investigador en el Departamento de Didáctica, Organización Escolar y Métodos de Investigación de la Universidad de Vigo, España. Dirección electrónica: mariabeatriz.paramo@uvigo.es

${ }^{3}$ Desarrolla su labor docente en el Jardim S. Vicente de Paula №1, Vila Real, Portugal. Dirección electrónica: genanecho@gmail.com
}

Ensayo recibido: 21 de abril, 2014

Enviado a corrección: 26 de setiembre, 2014

Aprobado: 8 de diciembre. 2014 


\section{Introducción}

\subsection{Desde una utopía a una realidad hacia la inclusión en Portugal}

El valor otorgado a la educación en estos últimos años ha ido variando en función de las concepciones sobre educación inclusiva regidas en cada momento en la sociedad y el sistema en el marco portugués. Resulta pertinente adentrarse en una perspectiva histórica que no se limite únicamente a especificar qué se hacía y qué no, sino también en ir presentando las diferentes características que definen la educación inclusiva en Portugal en función de diferentes textos legislativos, documentos, movimientos y asociaciones. El carácter de este análisis pretende enfatizar aspectos que en la actualidad podrían parecer, y podrían ser, contradictorios con los parámetros de la inclusividad y educación para el desarrollo; sin embargo, fueron concepciones e idearios necesarios y sucesivos para que actualmente se comprenda el sentido amplio de la educación inclusiva (Sanches y Teodoro, 2006).

La desavenencia manifestada en este contexto luso entre el carácter asistencial y educativo de la etapa comprendida de los 3 a 6 años de edad fue en su día el primer ingrediente del entramado teórico que se estaba configurando en forma de diferentes propuestas que abogaban por su inminente carácter educativo y por lo tanto, una modalidad integrada de todo el alumnado (Esteves y Lópes 2005). No obstante, el sistema educativo en Portugal se define por unas características históricas y sociales que lo limitan y delimitan, y en este caso fueron interpretadas como barreras. En el sentido amplio, por educación inclusiva nos referimos a "una escuela para todos" (Almeida y Alberte, 2009). El hecho de que teorías, autores y prácticas a lo largo de la historia se replanteasen esa educación ordinaria para todos, actualmente nos sitúan en un punto de cierta incertidumbre.

En los últimos años se ha visto la educación especial como un sistema educativo paralelo o separado del proporcionado a la mayoría de las niñas y niños, y este sistema ha sido desafiado por las ideas de la inclusión, en la cual se considera que todas y todos los niños deben formar parte del mismo sistema educativo (Florian, 2013). En el contexto portugués, la educación especial contaba con un corpus y un status propio que ya se desplazaba hasta el centro ordinario para dar respuesta a diferentes dificultades y necesidades que iban surgiendo. En este sentido, se puede mencionar la dimensión estructural, sin que ésta sea el único factor a tener en cuenta, sin embargo, constituye el primero de ellos, y el más complejo en este campo seguía siendo la dimensión social (Morgado, 2010). 
Con la formulación de muchas de las premisas de los Derechos Humanos, el desarrollo holístico empezaba a inundar los idearios de muchos docentes y expertos, y ya de algunas familias que concebían el sentido de la comunidad educativa en forma de referentes o apoyos. Estos dos principios originaron los primeros cambios legislativos en relación con la Educación especial, con proposiciones acerca de la adecuación de las condiciones en los centros ordinarios para niñas y niños que presentaran dificultades y necesidades, hasta abarcar términos de flexibilización curricular y organización de apoyos.

En la actualidad, según Sanches y Teodoro (2006), se ha constituido una línea recta que cruza el sistema educativo donde un primer punto sería el alumnado (que limita la Educación especial) hasta alcanzar el contexto (principio de la inclusividad). Las políticas educativas vigentes abogan por una educación ordinaria para todo el alumnado en las escuelas regulares siendo, de acuerdo con Rodrigues y Nogueira (2010), un 2\% de alumnos escolarizados en escuelas especiales. El Decreto-Lei 3/2008, de 7 de enero concreta muchos aspectos sobre esta temática. Por un lado, puntualiza y aclara el significado de Educación especial destinada a alumnado con Necesidades Educativas Especiales de Carácter Permanente (NEECP), su Plano Educativo Individualizado (PEI) y la diferencia del Apoyo Educativo, con el que se garantiza la atención a dificultades de aprendizaje.

Es en este documento legislativo donde se percibe un concepto más amplio de la inclusión, como un lugar de diversidad de necesidades, intereses y características (Freire, 2008). Uno de los principales logros fue entender el Jardín de Infancia (etapa educativa en Portugal que comprende la edad preescolar de los 3 a 6 años) como espacio de flexibilización, observación y detección para dar respuesta educativa a la participación del estudiantado, sin importar sus condiciones. El énfasis se encuentra en el concepto de inclusión. Si desde los inicios se cimientan características inclusivas en los diversos niveles del sistema educativo nos podríamos encontrar con un compromiso educativo y social sin necesidad de apelativos conceptuales de inclusión.

Son numerosos los trabajos y aportaciones teóricas centrados en ofrecer cifras de alumnas y alumnos que presentan algún tipo de NEECP en las aulas portuguesas, junto con la relación de ventajas y obstáculos que se encuentra el personal a la hora de favorecer la integración de esta. No obstante, toma fuerza el que cada vez más contemos con trabajos que aportan definiciones sobre el concepto de inclusión (Acedo, 2008; Blanco, 2008; Franco, 2011; Torres, Lissi, Grau, Salinas, Silva y Onetto, 2013), y sobre los diferentes agentes implicados (Murillo y Duk, 2011).Desde el papel que se le ha otorgado a la escuela y la 
respuesta a las muchas y diferentes necesidades del alumnado acaecidas en el aula ésta conforma un ambiente donde se verifica la influencia de diferentes agentes.

Para que los avances en materia legislativa se puedan llevar al contexto real y concreto es necesario reunir esfuerzos para identificar y analizar elementos mediadores para convertirlos o hacerlos inclusivos (Franco, 2011). La implicación de todos los agentes responsables en este proceso (alumnado, familia, docente y comunidad) se conforma en un proceso de acción-reflexión-acción continua, tal y como presentan Rodrigues y Nogueira (2011): desde el alumnado, con y sin necesidades educativas especiales pero todos ellos y ellas con características, habilidades, limitaciones y potencialidades propias; hasta la comunidad, entendida como un conjunto de relaciones entre la dimensión física, funcional, relacional y temporal de las personas.

En definitiva, lo que supone la educación inclusiva es variado e impreciso en estos agentes (Correia, 2010), e incluso, aún en los diferentes niveles del sistema. Freire (2008) presenta un elemento clave en un primer nivel de reflexión y análisis de contextos inclusivos, al referirse a las creencias, de ahí que sea necesario partir del modelo teórico e ideológico del que emergen unas u otras prácticas docentes - en este caso concreto, en el contexto de Jardín de Infancia - y que tienen relación con la educación inclusiva. La tendencia en el resto de Europa se concentra en avances legislativos y redes de interacción. Concretamente, en las aulas portuguesas esto se plasma en proyectos de innovación e investigación no sólo centrados en aspectos educativos de gestión curricular, sino también en índices de ambiente de aprendizaje, de relación e interacción (Franco, 2011). Estos aspectos constituyen los pilares que sustentan el ensayo que nos ocupa.

\section{Desarrollo del tema}

\subsection{Proposición}

Este trabajo constituye una investigación de corte cualitativo e interpretativo (Hernández, Fernández y Baptista, 2010) con un marcado carácter descriptivo y exploratorio, basada en un estudio de caso de un sujeto. Supone un proceso de investigación de realidad única centrada en conocer y valorar las percepciones acerca de la inclusión realizada por una maestra de un Jardín de Infancia, ubicado en Vila Real (Portugal) en relación con los agentes participantes (alumnado, familia, docente y comunidad). Como objetivos específicos se plantean: 
- Identificar qué concepción se tiene de la educación inclusiva, sus implicaciones y los agentes que intervienen en un proceso de enseñanza-aprendizaje inclusivo.

- Indagar la puesta en práctica de la educación inclusiva teniendo en cuenta la documentación legislativa, de centro y de aula.

- Conocer la percepción de las diferentes dimensiones del ambiente como potenciadores de una educación inclusiva: espacios, actividades, tiempos y relaciones.

- Contrastar las percepciones que se tiene de la educación inclusiva con respecto a cómo se implementan.

Para la selección de la muestra del estudio de caso único, los criterios que se han tenido en cuenta han sido:

- Profesorado en activo en aulas de Jardín de Infancia en Vila Real (Portugal) ${ }^{4}$.

- Profesorado de aula mixta (alumnado con edades entre los 3 y 6 años de edad) donde se atiende a la diversidad en cuanto a estilos, características, etc.

- Profesorado con implicación activa en la metodología de proyectos.

- Profesorado inmerso en proyectos de innovación o de investigación, sean estos en relación con otros centros educativos de Vila Real, con la Universidad o con asociaciones de la ciudad.

- Profesorado comprometido con la educación inclusiva.

- Profesorado con voluntad en la participación del presente estudio.

De esta forma, la muestra queda conformada por una maestra cuya docencia es impartida en un Jardín de Infancia, el centro educativo es de carácter público y se encuentra ubicado en la ciudad de Vila Real (Portugal). La docente cuenta con 30 años de experiencia docente, el tamaño medio de su clase es hasta la fecha de 25 niñas y niños aproximadamente, de edades comprendidas entre los 3 y los 6 años de edad.

La evaluación, observación y reflexión (Morgado, 2009) de los aspectos y objetivos citados se llevó a cabo con cinco instrumentos distintos pero complementarios entre sí: entrevista semi-estructurada, escala de observación, documentos, materiales audiovisuales y notas de campo. Explicamos cada uno:

\footnotetext{
${ }^{4}$ Somos conocedoras que la ciudad de Vila Real se caracteriza por ser un punto de referencia en el marco portugués en aulas de edades preescolares muy novedosas.
} 
- La descripción y análisis de las concepciones sobre la educación inclusiva se han realizado a partir de una entrevista semi-estructurada, que incluye tres apartados:

Un primer bloque denominado "Comprensión, definición y marco de inclusión" destinado a conocer la opinión de la encuestada en aspectos como el grado de familiarización y comprensión del término inclusión, el significado que para ella abarca dicho concepto y la opinión sobre los efectos de la legislación educativa en esta temática.

Un segundo apartado denominado "Implicaciones en la escuela y práctica docente" con el que se busca conocer la realidad del aula, casos y situaciones reales en este marco. Así se plantean cuestiones que tratan de recabar la opinión de la entrevistada en cuanto a la educación inclusiva producida en su centro y cómo se plasma en los documentos organizativos y curriculares de este y del aula. Asimismo, se recaba información sobre cómo se traduce la educación inclusiva en su aula, cuál es el planteamiento, metodología o práctica docente que desarrolla para favorecerla, cómo es una organización que se asiente en pilares inclusivos, cómo es el clima del aula que favorece la inclusión, cómo se planifican las actividades de aprendizaje, materiales y recursos tanto personales como materiales, desde esta perspectiva de una inclusión educativa y por último, cómo se lleva a cabo la evaluación acorde a la inclusión.

El último apartado "Agentes, necesidades y obstáculos" incluye aspectos en relación con la participación con otros agentes (escuelas, familia, sociedad, etc.) y con la Universidad en la medida de si estos favorecen propuestas de mejora en educación inclusiva sobre su formación inicial y continua, si en la actualidad se promueven ofertas de formación basadas en la inclusividad, qué barreras y obstáculos se podrían señalar para el avance hacia la inclusión así como las necesidades que se plantean desde el aula para hacer efectiva la educación inclusiva en el trabajo con el alumnado.

- La docente informante e investigadoras han observado el mismo aspecto pero en momentos diferentes utilizando una escala de observación, la cual se basa en la escala de observación del ambiente de aprendizaje de Iglesias (2008), incluye, además de unos primeros datos contextuales del centro, aula y práctica docente, 4 grandes bloques:

- "Dimensión física del ambiente como respuesta a la educación inclusiva", estructurada, a su vez, en cuatro grupos de ítems. El primero se refiere a la estructura u organización del aula en cuanto a espacios, zonas y puntos; un 
segundo acerca de la existencia y tipo de delimitación (fuerte, débil) entre esta organización; otro referido al carácter dinámico de las zonas, el cual cuestiona el tipo de desplazamiento y su motivo; y por último, la modalidad de acceso a las zonas.

- La "Dimensión funcional y tipo de actividades para favorecer la inclusión", incluye ítems referidos a conocer el planteamiento educativo del aula, el tipo de actividades llevadas a cabo dentro de ella y su orden en una jornada escolar, el tipo de zonas en el que se ejecutan y la polivalencia de las actividades.

- La "Dimensión temporal dedicada a dar respuesta a la atención a la diversidad", facilita el registro de observación sobre aspectos relacionados con el tiempo dedicado a diferentes tipos de actividades (libre; planificada; gestión, servicio y rutina; y de evaluación).Y finalmente,

- La "Dimensión relacional entre docente y alumnado e iguales", facilita que la observación se realice sobre los distintos tipos de agrupamientos constituidos en diferentes momentos y el tipo de control y participación por parte de la docente y entre el alumnado, acaba con un espacio para elaborar un boceto del plano de aula. Aun presentando este instrumento como una escala de observación, es cierto que reporta muchos datos relacionados con el ambiente ordinario del aula. Cuando se presentan las líneas del estudio se hace hincapié en el ambiente y en las diferentes dimensiones, solicitando datos relacionados con la educación inclusiva y su realidad en el aula. Además, existe un último apartado para otras observaciones en las que se recogen datos (ya dados o que quedaron por decir) en cuanto a espacios y tiempos destinados para la atención de Necesidades Específicas de Apoyo Educativo (NEAE) del estudiantado, al control y participación en momentos de trabajo con NEAE y referido a algún tipo de actividad, recurso destinado a este alumnado.

- Sobre los documentos y materiales audiovisuales, se solicitó a la docente que nos aportase documentación legislativa y de organización curricular del centro y aula, así como materiales audiovisuales (fundamentalmente fotografías y vídeos) que dispusiese sobre la temática de estudio. Por último, las investigadoras han realizado durante un trimestre diferentes registros, haciendo uso de notas de campo, que recopilaban datos sobre los documentos de aula, organización del aula, horarios, percepciones, 
observaciones y comentarios por parte de la docente y de las investigadores (familia, agentes, clima, afecto, etc.) y otras reflexiones derivadas del diálogo conjunto.

La triangulación de datos a partir de la información recabada con los cinco instrumentos (entrevista, escala de observación, documentos, materiales audiovisuales y notas de campo) le otorgan a la investigación una inmersión inicial con respecto a la presentación del estudio y conocimiento y primera toma de contacto con la maestra, con el alumnado y los espacios.

La recolección de información se realizó durante un trimestre y mantuvo la siguiente secuencia. Una vez presentadas las líneas básicas de la investigación a la docente informante, durante dos sesiones de la jornada escolar, se llevó a cabo una recolección de datos por parte de las investigadoras utilizando la escala de observación diseñada. A posteriori, en una tercera sesión, se aplicó la entrevista y en una cuarta sesión se le presentó la escala de observación para que ella la realizase. Al hacerlo con carácter oral se da la opción para que entre investigadoras y docente surjan nuevas concepciones y datos a debatir para favorecer una retroalimentación y reflexión conjunta (Fuertes, 2011). Por último debe indicarse que todo el proceso se contrasta con las notas de campo recabadas por la investigadora así como con las fotografías y vídeos aportados y documentación significativa del centro y aula.

El análisis de datos se efectuó mediante un análisis de contenido. Se identificaron los códigos y categorías por parte de las investigadoras de forma separada e independiente, se sistematizó la información y se procedió a su respectiva interpretación, se contrastaron los mencionados sistemas de categorías, a partir de los cuales se definió un sistema común que integra y redefine las categorías individuales de análisis. Este análisis se apoyó en el programa informático Maxqda.

\subsection{Resultados del estudio}

La dialéctica del discurso inclusivo transcurre sobre qué y cómo están haciendo los principales actores del cambio, los docentes. El objetivo pasa por hacer de la reflexión sobre los parámetros y desafíos de la escuela un espacio para conocer las percepciones acerca de la inclusión, paradigma del sistema educativo actual. Atendiendo a los objetivos planteados, agrupamos la información en dos grandes bloques: uno que aglutina aquella derivada de la idea de educación inclusiva; y otro, referido a las distintas dimensiones relacionadas con la mejora sobre la educación inclusiva. Se debe señalar que las citas transcritas y recogidas 
mediante la entrevista y la escala de observación estarán integradas en el texto y vendrán identificadas por letra en cursiva y entre comillas. Dado que la conversación transcurrió en lengua portuguesa, el relato de la docente ha sido traducido al español.

\subsection{Conceptualización de la inclusión}

El cambio entre la filosofía de integración y el paradigma de inclusión supuso, en un primer momento, un proceso de reflexión sobre el sistema educativo, y que ahora se torna hacia los docentes (Sanches y Teodoro, 2007). De acuerdo con esto, la maestra comenta que desde su punto de vista actualmente las y los docentes entienden lo que la palabra inclusión significa, y la describe de la siguiente manera:

"Inclusión es tener en cuenta no sólo a alumnado con necesidades educativas, sino a todos y cada uno de los niños y niñas, que son diferentes y llegan a esta aula para aprender siendo felices".

A pesar de comprender y estar familiarizada con el concepto, reconoce que en ocasiones "no sabemos ponerlo en práctica". La docente indica que "es una realidad a la que queramos o no, tenemos que afrontar"; desde el marco legislativo, pasando por los documentos del agrupamiento (según señalan Rodrigues y Nogueira [2011], la organización del sistema escolar queda conformada como una unidad escolar, un agrupamiento, formado por diferentes centros distantes entre ellos, lo que rentabiliza recursos y gestión) hasta los del aula, estos, presentan orientaciones teóricas sobre cómo hacer de las aulas ambientes inclusivos.

También señala que "donde hay que concretar la inclusión es en el aula, donde soy yo, la auxiliar y 25 niños y niñas de entre 3 y 6 años con sus familias; el estar hablando de características psicoevolutivas diferentes por los rangos de edad y variados y cambiantes estilos de aprendizaje nos descubre indudablemente la atención a la diversidad”. Esto sugiere que las características del alumnado serán lo que modifique la práctica docente. A este respecto, manifiesta que "es miedo, a veces, a hacerlo mal, pero no pasa nada, nosotras también aprendemos siempre, si no nos estancaríamos y la educación no lo permite, pero otras muchas veces es falta de formación".

A partir de las ideas expuestas por la docente se comparten lo expuesto por Morgado (2009) quien señala la necesidad de planes de formación permanente, planteamiento compartido por la docente, quien señala: "repercutan en mi práctica”. Una práctica que se 
nutre de la investigación, de la reflexión y de la acción, como un proceso cíclico que revalorice el papel de los docentes y las docentes en las primeras edades (Sanches y Teodoro, 2007) como agentes de inclusión porque tal como señala nuestra informante: "muy pocos saben qué estamos haciendo en inclusión, y les asombra lo que un aula de Educación Infantil puede albergar".

\subsection{Dimensiones inclusivas}

En las siguientes líneas se recogen las percepciones de la maestra sobre las distintas dimensiones relacionadas con la mejora sobre la educación inclusiva, que Iglesias (2008) expone: dimensión física (en cuanto a cómo los espacios se organizan para atender a una práctica educativa inclusiva); dimensión funcional (que recoge reflexiones en cuanto a momentos y actividades que favorezcan esta realidad); dimensión temporal (la organización de la jornada escolar que pueda o no favorecer prácticas inclusivas); y por último la dimensión relacional (en el sentido de qué tipo de relaciones entre profesorado y alumnado se dan y si favorecen que la educación inclusiva se instaure en ese aula).

A nivel de concreción curricular la maestra señala que "cada año nuestro proyecto de aula, mi labor como docente y la del auxiliar cambian para responder a las características concretas que presenta nuestro alumnado, así como también el espacio, las actividades...”. La suposición de todos los agentes y elementos del ambiente se configuran como motor de prácticas y clima inclusivos (Morgado, 2009). La función del ambiente, según disponemos del testimonio de la docente, "tiene un carácter inminentemente globalizador", al igual que la propia etapa y la práctica docente. La maestra señala reiteradamente que la inclusión no se crea de una u otra determinada manera, sino que es de por sí, ya que "el ambiente hace que la inclusión sea algo real del aula". No se trataría tanto de espacios, ni actividades, ni tiempos, ni relaciones específicas para responder a necesidades específicas, sino de un marco y ambiente general que con identidad propia responda a ello, "pues de lo contrario ya no estaríamos hablando de inclusión".

En cuanto al espacio, existe una correspondencia entre la respuesta de la maestra en la entrevista y las observaciones realizadas por las investigadoras. No existe ningún espacio destinado exclusivamente a la atención de necesidades educativas. El aula está organizada por rincones y una mesa central de trabajo con 14 sillas (téngase en cuenta que son 25 niños y niñas en el aula, lo que indica la forma en la que el espacio, los rincones y la gestión del aula ocupan). Existe una fuerte delimitación entre zonas y puntos del aula que según la 
encuestada "permiten trabajar en los distintas zonas simultáneamente con variedad de agrupamientos y finalidades específicas que ellos y ellas conocen".

Aun siendo así esa demarcación, la maestra señala que se produce un dinamismo en tanto al mobiliario y su disposición a lo largo del curso académico, un ejemplo de ello es la siguiente afirmación: "las necesidades de hoy, pueden ser habilidades mañana y aparecer otras dificultades que nos obliguen a reestructurar el espacio"; también de actividades y movimiento del alumnado a lo largo de la jornada escolar. El sistema de acceso a los diversos espacios viene determinado por un pictograma en cada rincón de la cantidad ratio de niños y niñas que pueden permanecer en él, y un método de collares con la imagen del rincón para cada estudiante. La maestra lleva un registro diario de qué espacios y zonas visita cada niño o niña, tal como manifiesta:"para conocer sus muy variados intereses, el punto de partida para una educación personalizada, para conocer destrezas y fortalezas y poder orientar los siguientes días otro tipo de espacios para potenciar sus debilidades 0 limitaciones". Es preciso señalar que en caso de alguna NEECP, el rol de la docente es "observar e investigar desde el juego y la creatividad (lo que Sanches y Teodoro [2006] denominan la capacidad de improvisación de un maestro/a, capacidad y factor esencial en la escuela inclusiva), para poder intentar comprender que es lo que está pasando para solicitar después la valoración del psicólogo del agrupamiento" y en caso de ser necesario, los docentes especialistas del agrupamiento: "visitarían el aula y trabajarían in situ con el niño o niña, aquí con todos nosotros y con actividades que partan de nuestro proyecto común".

De acuerdo con Esteves y Lópes (2005), es necesario señalar que existen ciertas barreras arquitectónicas que podrían dificultar construir espacios inclusivos, existiendo escuelas de referencias que trabajaron en esos obstáculos (Rodrigues y Nogueira, 2011), porque en numerosas ocasiones, según nos señala la docente: "tenemos que lidiar con aspectos que dificultan la inclusión, ajenos a nosotros, pero vamos resolviendo...”.

La organización del espacio físico determina la funcionalidad del ambiente en unos $u$ otros momentos y actividades que, en criterio de la informante "no necesitarían la palabra inclusiva". La disposición en rincones y pequeños grupos de trabajo posibilita la cooperación entre la docente y la auxiliar de manera que la atención es individualizada ya que según indica "me permite trabajar de manera personalizada con todos y todas en sus destrezas $y$ posibles limitaciones mientras la auxiliar guía los otros grupos”. Las observaciones por parte de la investigadora y las actividades y momentos relatados por la maestra constatan la semejanza y paridad entre éstos, que contemplan pequeñas adaptaciones y variabilidad de 
finalidades y capacidades. Se destaca que los recursos utilizados son también homogéneos para todo el estudiantado, especificando que "son ellos quienes lo hacen heterogéneos e inclusivos buscando formas y usos", la maestra verbaliza y muestra gran variedad de proyectos y actividades en formato tecnológico que responden a una educación inclusiva.

También existe material específico para algún alumno o alguna alumna brindado por el personal especialista que trabaja con él o ella, a modo que posibilite que la maestra comprenda cómo se está procediendo y en qué términos, ello, para hacer de la educativa algo transversal también en el aula. Sólo se evidencia la existencia de libros del alumnado para las áreas de la lengua escrita y de la matemática, el resto de material didáctico lo elabora la maestra y responde a los términos de inclusividad, según nos señala: "a partir de las consideraciones del proyecto en cuanto a capacidades y objetivos, modificando la forma y contenido según lo que nos encontremos en el aula en ese momento determinado".

Un punto a destacar es la importancia que desde esta aula se le da a las actividades y momentos de evaluación (Morgado, 2010). El aprender a aprender y la evaluación del proceso de enseñanza-aprendizaje conformarían el marco de una educación inclusiva (Sáiz, Flores y Román, 2010). En este sentido, "crear los materiales de evaluación supone partir de las características de cada niño y niña, de su proceso, de sus cambios y de sus mejorías, conocerlo, interpretarlo y modificar mi práctica y el ambiente en función de ello". La maestra y la auxiliar realizan actividades de evaluación de manera personal con cada alumna o alumno, por medio de de tablas de doble entrada que se archivan en su correspondiente expedientes (estrategia inclusiva que presentan Rief y Heimburge, [2000]), registros y paneles y carteles que inundan el aula para reflejar qué se hizo, cómo, valoraciones, destrezas mejoradas, habilidades por adquirir o mejorar y cómo, etc.

Recogemos la siguiente afirmación relacionada con este aspecto:

"Crear los materiales de evaluación supone partir de las características de cada niño y niña, de su proceso, de sus cambios y de sus mejorías, conocerlo, interpretarlo y modificar mi práctica y el ambiente en función de ello", utilizando para ello "tablas y más tablas para evaluar de todo y con cada niño y niña, es un trabajo muy latoso y lento, pero merece la pena centrarse en cada uno y aprender de esta manera".

La gestión del tiempo de la jornada escolar (Morgado, 2009) puede esclarecer y mostrar cómo se distribuye el tiempo para trabajar con diferentes necesidades, dificultades o habilidades a impulsar. La maestra centra parte de sus esfuerzos en distribuir la jornada 
escolar en momentos de gestión, servicios y rutinas, según sus palabras no son más que “oportunidades para designar cada día encargados y encargadas de aula que tendrán que aprender a ser conscientes y comprender sus labores, pero también qué pueden y saben hacer los otros y que deben mejorar"); y momentos de planificación del proceso de enseñanza aprendizaje para desarrollar los recursos de cognición propios para el alumnado (Sanches y Teodoro, 2006) en cuanto a qué vamos a hacer, cómo, por qué y para qué. Esto lo explica así: "es como dar paso a aprender a aprender", a atender a la diversidad desde la autonomía del alumnado (Santos y César, 2010), "es lo que yo entiendo de inclusión y cómo yo lo llevo a mi práctica y aula”. El poder contar con la presencia de otra persona en el aula, en este caso la asistente, aumenta las posibilidades de gestionar y destinar intervalos de tiempo específicos para trabajar algo concreto de manera personalizada y reflexiva (Morgado, 2009); al mismo tiempo que se está desarrollando la autonomía en la niñez del resto del grupo, capacidad que sustenta la inclusión en la actualidad.

La construcción de nuevas realidades inclusivas es el resultado de un trabajo conjunto de optimizar las relaciones (Rodrigues y Nogueira, 2011). Al aplicar los instrumentos, subyacieron diversas tipologías y creencias sobre las relaciones que fomentan un proceso educativo inclusivo que dibuja una línea recta entre alumnado, aula, escuela y comunidad. "Son ellos y ellas quienes tienen que aprender los unos de los otros"; creencia que demuestra la importancia de la tutoría entre iguales (Santos y César, 2010) y del aprendizaje cooperativo para que la educación inclusiva sea un concepto y un valor que parta de y sobre ellos y ellas.

La estructura del ambiente de aprendizaje posibilita que el control y participación que el alumnado hace sobre su propio aprendizaje sea elevado, acompañado de un control indirecto y una participación puntual en momentos de actividad dirigida por parte de la docente y asistente. Estos dos últimos protagonistas y agentes de la educación inclusiva necesitan de una coordinación, "nos acoplamos las dos al trabajo de la otra, sabiendo cómo trabaja cada una, que es lo que se quiere y cómo hacer frente a situaciones de atención a la diversidad y necesidades", junto también con las personas que participan como animadores del centro (esta figura se corresponde con auxiliares que están con el alumnado en las horas previas a comenzar la jornada escolar, durante la comida y las actividades después de finalizar el horario lectivo). Estas personas comparten percepciones, analizan, observan e intervienen en términos de inclusión con el resto de maestras y auxiliares del jardín y con las personas especialistas del agrupamiento, manteniendo reuniones periódicas. 
En cuanto a las relaciones con la familia, la docente afirma que "hay una relación que va a favorecer o dificultar el poder poner en práctica claves inclusivas: la familia". La ley 21/2008, de 12 de Maio (Diário da República Portuguesa no 91, I Série), que modifica la ley anteriormente citada, enfatiza la participación de las familias y un trabajo colaborativo como agentes de cambio hacia el respeto por la diferencia y la diversidad (Susinos y Rodríguez, 2010), siendo las propias familias las que escogerán la modalidad de escolarización en caso de necesidades específicas. La entrevista individual inicial que la maestra ha realizado a comienzo de curso a los padres y madres de familia del grupo para indagar sobre intereses, posibilidades y destrezas. La hoja de registro de posibles incidentes, el listado de elementos y trabajos que las familias aportan al aula, así como un análisis de contenido de las entradas publicadas en el blog de aula revalida el convencimiento de la docente hacia la importancia que tiene su papel:

"A lo largo del año tenemos muchas reuniones con las familias, pero es importante, tienen que saber qué materiales necesitamos para llegar a todos y cada uno de los niños/as, qué estamos haciendo y para qué y de manera indirecta hacerles llegar valores de respeto a las dificultades".

Más allá de la familia, la comunicación constante entre los distintos profesionales que trabajan con el escolar o la escolar constituye un sentido muy amplio de comunidad educativa, facilitando procesos de reflexión y acción conjuntos (Sanches, 2005). Ejemplo de ello es el siguiente fragmento:

"Desde los pediatras, logopedas, psicólogos parecen estar bajo el mismo paraguas, pues nos hacen llegar al aula sus impresiones y percepciones sobre dificultades $y$ mejorías".

Y por último, en cuanto a las relaciones con los centros de formación del profesorado, la encuestada manifiesta la relación que debe existir entre universidad y escuela en temas de inclusión "tendría que ser mucho más sólida, para que la teoría no sufriera desfase con la práctica"; "si la universidad y la investigación viniesen a las aulas, supondría un soplo de aire fresco para nosotras". Los postulados teóricos y conceptuales de la educación inclusiva parecen situarse en el discurso de la maestra, desde la práctica e intervención real en las aulas. En definitiva, escuela y Universidad "debieran trabajar juntos en ellos (refiriéndose a los postulados)". 


\section{Conclusiones}

Este ensayo ha permitido conocer qué percepciones y reflexiones hay en torno a la inclusión desde los ojos de una maestra. En este caso, las concepciones que priman han sido configuradas en categorías inclusivas de análisis y cambio, entre las cuales se observan: concepciones, realidades, necesidades, dimensión física del ambiente, funcionalidad de la inclusión, estructura temporal y las relaciones.

El indagar e identificar las concepciones acerca de la educación inclusiva, de sus implicaciones y los agentes que intervienen en el proceso de enseñanza y aprendizaje inclusivo (objetivo 1) permite conocer necesidades, obstáculos y miedos, pero también oportunidades de aprendizaje que, si se dan a conocer, puedan repercutir en el quehacer de otros maestros y maestras (Sanches, 2005). Por otro lado, son necesarias más muestras y difusión de estas prácticas y concepciones, e igualmente, conocer e indagar sobre ellas teniendo en cuenta el marco curricular y legislativo en el que se soportan (objetivo 2). La palabra equidad empieza a emerger en muchos discursos y prácticas (Freire 2008), pero cabría preguntarse si es así en los diferentes textos legislativos y documentos de centro y aula porque la educación inclusiva no consiste únicamente en que todas y todos estén en una misma aula, sino en un proceso educativo de calidad para todos ellos y ellas que tiene su primera concreción en todos estos documentos.

Se entiende como algo natural que los espacios, actividades, tiempos y relaciones respondan a un conjunto de elementos que, según unas u otras relaciones, se configuran para hacer efectiva la realidad inclusiva en el aula. Este primer análisis de dimensiones y claves inclusivas del ambiente de aprendizaje (objetivos 3) supuso una reflexión acerca de la dimensión física del ambiente de aula, en el que su estructura, delimitación, carácter y acceso puede situarnos ante espacios de por sí inclusivos y que atiendan a la diversidad.

Acerca de la dimensión funcional, en la que la secuencia de actividades de diversas índoles y a su vez simultáneas, atienden a la dimensión temporal, favorece una atención más individualizada y un abanico más amplio de posibilidades; y de la dimensión relacional, en la que nos aventuraríamos a seguir las palabras de la maestra de que un aula mixta se beneficia de principios inclusivos y de atención a la diversidad.

Triangular esta información recabada por los diferentes instrumentos permite constatar las percepciones que esta maestra tiene de la educación inclusiva y su implementación real en las aulas (objetivo 4). La reflexión acerca de la capacidad que la docente tiene de estructurar y movilizar los diferentes elementos que configuran el ambiente nos sitúa ante 
unas primeras claves inclusivas en relación con autores y corrientes inclusivas actuales que aún no pudiendo generalizarse, por situarnos ante un estudio de caso, debe contribuir y contribuye, a una reflexión sobre la reconstrucción de las concepciones de los profesores a la luz del actual sistema educativo. Conscientes de esta limitación, la prospectiva de investigación pasaría por aumentar la muestra, lo que podría acercar datos y un mapa de prácticas educativas inclusivas sobre lo que supone la educación inclusiva en las aulas portuguesas, qué tipo de prácticas conlleva y los valores docentes determinadas por sus concepciones (Freire, 2008).

En una educación del desarrollo, y por lo tanto en una sociedad del desarrollo, las competencias son un elemento clave para nuestro alumnado, pero también lo son para nuestro desarrollo personal (Correia, 2010). Por lo tanto, la inclusión no deja de ser una oportunidad de capacidad docente (Morgado, 2009), de plantearnos retos y desafíos. Tal como Almeida y Alberte (2009) afirman, este tipo de trabajos podrían ser un elemento revitalizador que potencie la inclusión del alumnado.

\section{Referencias}

Acedo, Clementina. (2008). Educación inclusiva: superando los límites. Perspectivas. Revista Trimestral de Educación Comparada, 38(1), 5-16.

Almeida, Magda Sofía de y Alberte, José Ramón. (2009). Las concepciones de los profesores y la respuesta a la inclusión en Lisboa. Revista de Educación Inclusiva, 2(2), $\quad 1-11 . \quad$ Recuperado http://www.ujaen.es/revista/rei/linked/documentos/documentos/3-2.pdf

Blanco, Rosa. (2008). Construyendo las bases de la inclusión y la calidad de la educación en la primera infancia. Revista de Educación, (347), 33-54. Recuperado de http://www.revistaeducacion.mec.es/re347/re347 02.pdf

Correia, Luis (coord.). (2010). Educaçao Especial e Inclusão. Quem disser que uma sobrevive sem a outra não está no seu perfeito juízo. Porto: Porto Editora.

Esteves, Alberto y López, Manuel. (2005). La integración educativa en Portugal. Un análisis de la enseñanza obligatoria. Revista Española de Educación Comparada, (11), 315356. Recuperado de http://www.sc.ehu.es/sfwseec/reec/reec11/reec1111.pdf

Florian, Lani. (2013). La educación especial en la era de la inclusión: ¿El fin de la educación especial o un nuevo comienzo? Revista Latinoamericana de Inclusión Educativa, 7(2), 27-36. Recuperado de http://www.rinace.net/rlei/numeros/vol7-num2/art1.pdf

Franco, Vítor. (2011). A inclusão começa em casa. En David Rodriguez, Educação Inclusiva - Dos conceitos às práticas de formaçãok (pp. 157-170). Lisboa: Instituto Piaget. 
Freire, Sofía (2008). Um olhar sobre a inclusão. Revista de Educaçao, 16(1), 5-20. Recuperado http://repositorio.ul.pt/bitstream/10451/5299/1/Um\%20olhar\%20sobre\%20a\%20Inclus\% C3\%A30.pdf

Fuertes, M $\mathrm{M}^{\mathrm{a}}$ Teresa. (2011). La observación de las prácticas educativas como elemento de evaluación y de mejora de la calidad en la formación inicial y continua del profesorado. Revista de Docencia Universitaria, 9(3), 237-258. Recuperado de http://redu.net/redu/documentos/volumenes completos pdf/vol9 n3 completo.pdf

Hernández, Roberto, Fernández, Carlos y Baptista, Pilar. (2010). Metodología de la investigación. México: Mc Graw Hill.

Iglesias, Ma Lina. (2008). Observación y evaluación del ambiente de aprendizaje en educación infantil: dimensiones y variables a considerar. Revista lberoamericana de Educación, 47, 49-70. Recuperado de http://www.rieoei.org/rie47a03.pdf

Morgado, José. (2009). Educação inclusiva nas escolas actuais: contributo para a reflexão. En Bento Silva; Leandro Almeida; Alfonso Barca; Manuel Peralbo, Actas do $X$ Congresso Internacional Galego-Português de Psicopedagogia (pp. 104- 117). Braga: Centro de Investigação em Educação (CIEd)-Universidade Minho.

Morgado, José. (2010). Os desafíos da Educación Inclusiva: fazer as cousas certas ou fazer certas as cousas. En Luis Correia (Coord.), Educaçao Especial e Inclusão. Quem disser que uma sobrevive sem a outra não está no seu perfeito juízo (pp.73-88). Porto: Porto Editora.

Murillo, Francisco Javier y Duk, Cynthia. (2011). ¿Escuelas eficaces versus inclusivas? Revista Latinoamericana de Educación Inclusiva, 5(1), 11-12.

Portugal. (7 de Janeiro, 2008). Decreto-Lei nº 3/2008. Diário da República Portuguesa, nº 4, I Série.

Portugal. (12 de Maio, 2008). Lei no 21/2008. Diário da República Portuguesa, no 91, I Série.

Rief, Sandra y Heimburge, Julie. (2000). Como ensinar todos os alunos na ala de aula inclusiva. Porto: Porto Editora.

Rodrigues, David y Nogueira, Jorge. (2010). Educaçao Especial e Inclusiva em Portugal. Factos e opções. Revista Educación Inclusiva, 3(1), 97-109.

Rodrigues David y Nogueira, Jorge. (2011). Educação Especial e inclusiva em Portugal: fatos e opções. Revista Brasileira de Educação Especial, 17(1), 3-20. Disponible en: http://www.scielo.br/scielo.php?script=sci arttext\&pid=S1413-65382011000100002

Sáiz, Ma Consuelo, Flores, Valle y Román, José María. (2010). Metacognición y competencia de "aprender a aprender" en Educación Infantil: una propuesta para facilitar la inclusión. Revista Electrónica Interuniversitaria de Formación del Profesorado, 13(4), 123-130.

http://aufop.com/aufop/uploaded files/articulos/1291992801.pdf 
Sanches, Isabel. (2005). Compreender, Agir, Mudar, Incluir. Da investigação acção è educação inclusiva. Revista Lusófona de Educação, 5, 127-142. Recuperado de http://www.scielo.oces.mctes.pt/pdf/rle/n5/n5a07.pdf

Sanches, Isabel y Teodoro, António (2006). Da integração à inclusão escolar: cruzando perspectivas e conceitos. Revista Lusófona de Educação, 8, 63-83. Recuperado de http://www.scielo.gpeari.mctes.pt/pdf/rle/n8/n8a05.pdf

Sanches, Isabel; Teodoro, António (2007). Procurando indicadores de educação inclusiva: as práticas dos professores de apoio educativo. Revista Portuguesa de Educação, 20(2), 105-149. Recuperado de http://www.scielo.oces.mctes.pt/pdf/rpe/v20n2/v20n2a05.pdf

Santos, Joel y César, Margarida. (2010). Atitudes e preocupações de professores e outros agentes educativos face à incluso. Interacções, 6(14), 156-184. Recuperado de http://revistas.rcaap.pt/interaccoes/article/view/419

Susinos, Teresa y Rodríguez, Carlos. (2011). La educación inclusiva hoy. Reconocer al otro y crear comunidad a través del diálogo y la participación. Revista Interuniversitaria de Formación del Profesorado, 70(1), 15-30.

Torres, Natalia, Lissi, María Rosa, Grau, Valeska, Salinas, Marcela, Silva, Mariela y Onetto, Valentina. (2013). Inclusión educativa: componentes socio-afectivos y el rol de los docentes en su promoción. Revista Latinoamericana de Inclusión Educativa, 7(2), 159173. Recuperado de http://www.rinace.net/rlei/numeros/vol7-num2/art9.pdf 